\title{
Grado, clase y privilegio en la formación de doble grado en cirugía maxilofacial
}

\author{
Degree, class and privilege in dual-degree maxillofacial surgery training
}

\section{Sr. Editor:}

La especialidad de Cirugía Maxilofacial (CMF) presenta particularidades en sus vías de formación, dependiendo del país, se exige el doble grado (médico y dentista), solo médico o solo dentista (1). La discusión técnica si el CMF necesita ser médico para tener las competencias para acceder al "quehacer complejo" de la especialidad ha sido tema recurrente de discusiones, estudios y editoriales en las revistas más importantes de la especialidad, sin llegar a una conclusión aceptada transversalmente $(1,2)$. Sin embargo, desde un punto de vista político existen una serie de cuestiones que, a mi parecer, tienen un profundo impacto en el cómo se podría implementar a nivel curricular el doble grado en Latinoamérica, impactando así en lo técnico. Lo anterior, se resume en la tríada de niveles: grado, clase y privilegio.

En el primer nivel del grado es importante señalar que si bien los primeros 2 años de medicina y odontología comparten cierta proporción de contenidos biomédicos fundamentales (anatomía, biología, fisiología, farmacología y otras), en esos primeros 2 años las mallas curriculares ya presentan diferencias. Como punto de comparación, en Norteamérica, casi la totalidad de los estudiantes de odontología y medicina han completado 4 años previos de College cursando los contenidos biomédicos fundamentales ya mencionados. De vuelta en Latinoamérica, a partir del tercer año, los contenidos de ambas carreras se diferencian aún más, por lo que, un dentista no cuenta con la formación médica de 5 años, incluyendo los 2 años de internado. Siguiendo en Latinoamérica, al término de los 5 a 7 años de universidad, médicos y dentistas tenemos la potestad para ejercer la profesión a nivel general de manera totalmente independiente. Esto difiere de otros países con carreras de medicina más cortas, incluso de 3 años en Norteamérica, donde el médico titulado debe realizar 2 o 3 años de postgrado (supervisado) antes de tener una licencia para ejercer independientemente (3).

En un segundo nivel, en Latinoamérica los cirujanos tienen una formación de base en Cirugía General (CG) de 2 a 4 años para luego acceder a la subespecialidad. Vale decir, existe una clase de especialistas médicos que denominamos cirujanos y que comparten el haber cursado de 2 a 4 años de cirugía general luego de haber terminado la carrera de medicina. Más aun, con estos 2 o más años, no nos referimos solo al cumplimiento de ciertos requisitos, sino a un conjunto de experiencias formativas compartidas por un grupo que determinan una visión del quehacer quirúrgico y un sentido de pertenencia a esa clase. Por tanto, desde esta visión de clase, la formación del CMF con doble graduación debería considerar esos 2 años de formación en CG.

En el tercer nivel del privilegio, recordar que el quehacer quirúrgico complejo en territorio 
craneofacial es compartido por las especialidades de Cirugía de Cabeza y Cuello, Cirugía Plástica y Reparadora, y Otorrinolaringología. Esto implica que, los especialistas en CMF doble grado deberían tener un periodo de formación compartida con alguna o todas esas especialidades, periodo que probablemente debería ser de al menos 1 año. Esta validación ante pares, a diferencia del grado y la clase, que están estrictamente relacionados con el respaldo legal, apuntan a que clínicas y hospitales se guardan el derecho a discreción de aceptar o no que un determinado profesional realice un determinado procedimiento en sus instalaciones. Este privilegio es la última milla para acceder efectivamente al "quehacer complejo".

Así, sería del todo preocupante asumir una visión simplista de la formación de doble grado en CMF para Latinoamérica, reduciéndola a un trámite de un par de años con el mero objeto de contar con el respaldo legal del título médico como algunos pretenden (4). Dicha aspiración está muy alejada del espíritu que marcó la introducción de los programas de doble grado en Norteamérica (2) de la mano del Dr. Guralnick en 1971 desde Harvard, quien identificó 4 ideas fuerza: existe un déficit educativo en los programas de CMF en cuanto a antecedentes médicos y quirúrgicos generales; esa deficiencia se corregirá mejor mediante la obtención de un título de médico y capacitación en cirugía general, además de la experiencia en CMF; ello es consistente con la dirección de la especialidad y estándares de formación; y, no se puede hacer un cambio curricular importante sin un trauma considerable para el grupo afectado por él. Estas 4 ideas son totalmente pertinentes a la realidad latinoamericana, por lo que deben ser consideradas a la hora de generar propuesta para la formación de especialistas en CMF con doble grado.

\section{Ricardo Cartes-Velásquez ${ }^{1, \mathrm{a}}$}

${ }^{1}$ Universidad Andrés Bello. Concepcion, Chile.

${ }^{\text {a }}$ Profesor Asociado ; DDS PsyD MD MPH PhD

\section{Correspondencia:}

Dr. Ricardo Cartes-Velásquez.

Correo electrónico: cartesvelasquez@gmail.com

\section{REFERENCIAS BIBLIOGRAFICAS}

1. Cartes-Velásquez R, Ramírez H. Doble Graduación en Cirugía Oral y Máxilofacial. Int J Odontoestomat. 2011;5(2):191-8.

2. Donoff B, Troulis MJ. The Development of DualDegree Programs in Oral and Maxillofacial Surgery: Celebrating the 75th Anniversary of the Journal of Oral and Maxillofacial Surgery and the 100th Anniversary of Our Specialty. J Oral Maxillofac Surg. 2018;76(6):1147-1149.

3. Kaban LB, Perrott DH. Dual-degree Oral and Maxillofacial Surgery Training in the United States: "Back to the Future". J Oral Maxillofac Surg. 2020;78(1):18-28.

4. Ramírez H, Vargas Á, Goñi I, Rosa A. Caracterización de programas de formación de especialistas en cirugía maxilofacial en Estados Unidos, Canadá y Chile. Rev Esp Cir Oral Maxilofac. 2020:42(1):40-6.

Recibido: 07-03-19

Aceptado: 23-06-20 